\title{
Formation des enseignants : un exemple de recherche-action: Chypre, France, lrlande, République tchèque, Slovénie
}

Training science teachers: an example of research-action Selected data from a European project: Cyprus, France, Ireland, Czech Republic, Slovenia Formación de los profesores de ciencias: un ejemplo de investigación-acción Algunos datos procedentes de un proyecto europeo: Chipre, Francia, Irlanda, República checa, Eslovenia

Groupe interuniversitaire projet Sophia

\section{(2) OpenEdition} Journals

Édition électronique

URL : http://journals.openedition.org/ries/660

DOI : $10.4000 /$ ries. 660

ISSN : 2261-4265

Éditeur

Centre international d'études pédagogiques

Édition imprimée

Date de publication : 1 septembre 2009

Pagination : 47-58

ISSN : $1254-4590$

\section{Référence électronique}

Groupe interuniversitaire projet Sophia, «Formation des enseignants : un exemple de rechercheaction: Chypre, France, Irlande, République tchèque, Slovénie », Revue internationale d'éducation de Sèvres [En ligne], 51 | septembre 2009, mis en ligne le 01 septembre 2012, consulté le 30 avril 2019 URL : http://journals.openedition.org/ries/660; DOI : 10.4000/ries.660 


\title{
Formation des enseignants : un exemple de recherche-action
}

\author{
Chypre, France, Irlande, République tchèque, Slovénie
}

\author{
Groupe interuniversitaire \\ projet Sophia
}

\begin{abstract}
"Réformer l'éducation est un art particulièrement difficile; il est rempli de pièges parce qu'avant de réformer l'éducation, vous devez rééduquer les éducateurs». Karl Popper
\end{abstract}

Le travail de recherche présenté dans cet article s'inscrit dans le cadre d'un projet de recherche européen sur l'enseignement des sciences (projet COMENIUS) ${ }^{1}$, mené par cinq équipes basées à Chypre, en Irlande, en Slovénie, en République tchèque et en France ${ }^{2}$. Le projet, conduit sur trois ans, a débuté en septembre 2006 et porte sur l'articulation entre la formation des enseignants et l'action pédagogique en classe. L'équipe de chercheurs a élaboré des modules de formation à la recherche de modalités opérationnelles permettant d'engager les enseignants dans une démarche socio-constructiviste.

S'appuyant sur l'expertise des chercheurs engagés dans ce projet, cet article collectif présente la situation de l'enseignement des sciences dans les différents pays représentés et montre, au-delà d'une simple juxtaposition descriptive, comment notre groupe s'est trouvé confronté à un paysage contrasté. Nous cherchons à préciser quelques points saillants, à montrer similitudes et divergences des situations rencontrées, et à dégager quelques pistes concrètes d'action dans le domaine de la formation des enseignants.

1. Ce projet a reçu le soutien financier de la Commission européenne : Agence Éducation, Audiovisuel et Formation de l'Union européenne, programme Formation tout au long de la vie. L'article reflète les opinions de ses auteurs et n'engage pas la responsabilité de la Commission européenne.

2. Sont impliquées les universités suivantes : University of Cyprus à Nicosie pour Chypre, St Patrick's College à Dublin pour l'Irlande, Univerza $v$ Ljubljana pour la Slovénie, Zapadoceska Universita à Plzen pour la République tchèque, l’Université catholique de l'ouest pour la France, équipe IFUCOME. 


\section{L'ENSEIGNEMENT DES SCIENCES DANS LES CINQ PAYS}

\section{Entre curiosité et désenchantement}

Si l'on analyse les données chiffrées issues de plusieurs enquêtes menées en Europe à propos des sciences, de leur enseignement ou des représentations qui leur sont associées, le paysage qu'elles mettent à jour est contrasté. Les personnes interrogées manifestent curiosité et intérêt vis-à-vis de la science, mais il semble qu'au-delà des mots, les faits révèlent un «certain désenchantement». Les sciences suscitent un intérêt certain, mais celui-ci ne semble pas suffisant pour qu'un jeune souhaite faire carrière dans un domaine scientifique. Dans les cinq pays, les constats sont comparables.

Premièrement, on constate une baisse de l'appétence des jeunes pour les études scientifiques. D’une façon visible, ce désintérêt touche les choix de poursuite d'études à la fin de l'enseignement secondaire. Certaines filières scientifiques ou technologiques de l'enseignement supérieur semblent en déshérence. Les études et recherches consultées sont concordantes et mettent en évidence une tendance stable. La désaffection a été constatée dans les cinq pays étudiés : en République tchèque, l'on constate un déclin sur les quinze dernières années; en Irlande, le nombre de scientifiques requis pour les besoins de l'économie ne correspond pas à celui des "vocations scientifiques»; en France, la stabilité des effectifs de jeunes qui passent un bac S (scientifique) n'occulte pas le faible taux de poursuite d'études vers des disciplines scientifiques de l'enseignement supérieur; en Slovénie, les dernières données disponibles en 2009 confirment la chute du nombre de jeunes engagés dans ce type de disciplines (cette chute avait été constatée dans la décennie précédente). Par ailleurs et d'une façon plus discrète, d'autres indicateurs semblent pointer une tendance plus profonde. Les études menées dans des pays où il est possible de choisir des sujets d'études optionnels scientifiques indiquent que ce désintérêt se manifeste de façon assez précoce. En Slovénie par exemple, les chiffres révèlent que le nombre d'élèves qui choisissent un sujet scientifique optionnel à l'école primaire ou que le nombre d'élèves qui optent pour les sciences lors de leur examen terminal à la fin de l'enseignement secondaire décroît de façon constante $^{3}$, passant de $25 \%$ à $18 \%$ entre 1995 et 2003 . Ce constat apporte un éclairage sur d'autres données. Ainsi, en France le nombre important de lycéens qui conservent, à l'issue de leur seconde, des options scientifiques semble de bon augure en première analyse. On peut cependant s'interroger sur les raisons qui motivent ce choix. Ce choix ne résulte-t-il pas d'une vision pragmatique? L'image d'une «série d'excellence» qui donne accès à de larges opportunités est

3. RIC Report, National Exams Centre 2002/2003, Ljubljana : RIC 2003. 
associée à la série scientifique française et elle pourrait être prédominante lors de la décision d'orientation et du choix des options.

Au-delà des formes différentes que peut prendre ce phénomène, la vision d'ensemble suggère qu'il s'articule à un problème de fond, dont nous avons cherché à cerner quelques dimensions.

\section{S'agirait-il d'un faible pouvoir attractif des études scientifiques?}

Cette assertion est, semble-t-il, largement partagée dans l'opinion des cinq pays concernés par l'étude. Sur ce point, la situation varie sensiblement selon les disciplines scientifiques. Ainsi, certaines sciences du vivant sont en situation particulièrement difficile en République tchèque. C'est le cas de la botanique, mais aussi d'autres branches de la biologie, alors que dans le même temps, les études en sciences biomédicales demeurent attractives. Cette attractivité des études médicales ou biomédicales est un constat partagé.

«Faire des études scientifiques ne ferait plus recette!» Telle est la formule qui pourrait dépeindre la situation. Une série d'explications, relativement stable d'un pays à l'autre, lui est généralement associée. Sont alors convoqués le caractère abstrait des études scientifiques, leur trop longue durée ou leur trop grande difficulté en regard des perspectives de carrière et de salaires qu'elles ouvrent. Comment faire, alors, pour inverser la tendance et accroître le nombre d'étudiants en science et en technologie? Parmi les pistes d'action, on peut imaginer, comme l'a fait en 2006 le ministère de l'Enseignement supérieur slovène, d'accroître le nombre de bourses d'études destinées aux étudiants scientifiques ou de créer des conditions privilégiées pour favoriser les inscriptions dans les universités de sciences et de technologie. L'exemple slovène montre qu'on peut en attendre quelques résultats. Par exemple, le taux d'étudiants en mathématiques et en informatique, qui était de $7 \%$, est passé à $7,3 \%$ en $2006 / 07$ puis à $8,4 \%$ en $2008 / 09$. De pareilles mesures apportent une bouffée d'air bienvenue au système. Elles ont cependant un caractère un peu sporadique et des effets pervers peuvent en résulter. Ainsi, on a pu constater, à la suite de cette initiative, des tensions au sein même de l'Université slovène, les universités de sciences humaines et sociales dénonçant un privilège et réclamant la parité des mesures.

\section{Faut-il y voir un problème sociétal ?}

Dans ce cas quels en sont les fondements? Provient-il d'une perte de prééminence des sciences dans le champ du savoir au profit d'autres domaines? Provient-il de profondes mutations dans les aspirations des jeunes? Des éléments viennent à l'appui des deux thèses. 
À l'appui de la première, si les disciplines scientifiques, dans la première moitié du vingtième siècle, pouvaient prétendre à une certaine suprématie au sein du champ du savoir, l'avènement de la société de l'information et de la communication a bouleversé la donne. Actuellement, la prééminence des domaines d'études comme le commerce, la finance ou le management semble réelle et ces spécialités se présentent comme de puissants facteurs d'attractivité : les carrières qu'elles ouvrent incarnent un futur désirable en phase avec l'évolution des aspirations de la jeune génération. Ce point nous amène à considérer la deuxième thèse en présence, que des études permettent d'étayer.

Ainsi une série d'enquêtes conduites en Slovénie ${ }^{4}$ a mis en évidence la rupture qui s'est produite dans le vécu de la jeune génération. Les étudiants des années 2000 de ce pays ont connu l'âge d'or d'une enfance sans soucis vécue dans un cadre économique stable. Ils se trouvent confrontés aujourd'hui à un système d'étude devenu plus compétitif, à un marché du travail dont l'offre s'est restreinte, à un monde du travail dans lequel les relations se sont dégradées. Dans ce contexte, le passage à l'âge adulte se fait plus difficilement. Les études montrent que le trait caractéristique de cette tranche d'âge est la volonté de se construire une identité. Il s'agit "d'être»; "être» par exemple un étudiant. Les études sont l'un des moyens choisis par les jeunes pour se construire cette identité. Deux lignes de force partagent alors la jeunesse selon la façon dont les jeunes perçoivent ce qui doit être le pivot de leur identité. Pour «être», un premier groupe mise sur une identité sociale. Les études sont l'un des moyens de la construire. Les choix d'orientation intègrent les standards de la société actuelle et ce sont les études qui permettent d'envisager une belle carrière ou un haut statut social; la finance, l'économie ou le management ont alors la préférence. Un jeune peut aussi considérer que le point majeur dans la construction de son identité est l'épanouissement personnel. La demande d'études est alors autre : il s'agit pour le jeune de "trouver sa voie» et le choix se porte vers des domaines comme l'art ou les sciences humaines.

Entre ces deux lignes se trouve le territoire restreint des disciplines scientifiques car elles ne répondent stricto sensu à aucun des deux critères. En République tchèque, le même sentiment de rupture a été vécu. En France, une tendance de fond analogue aux constats slovènes existe, mais les analyses de B. Convert $(2006)^{5}$ montrent que ce phénomène touche l'ensemble des disciplines théoriques de l'Université. La «crise de l'emploi» aurait conduit à un «détournement des orientations des étudiants vers les formations professionnalisées", les études les plus convoitées seraient celles qui offrent «les diplômes les plus facilement monnayables sur le marché de l'emploi».

4. EURYDICE, L'enseignement des sciences dans les établissements scolaires en Europe. Etat des lieux de la politique et de la recherche, 2006. Voir : http://www.eurydice.org/.

5. CONVERT, B. (2006). Les impasses de la démocratisation scolaire. Sur une prétendue crise des vocations scientifiques. Paris : Editions Raisons d'agir. 


\section{S'agit-il de la façon dont les sciences sont enseignées?}

La lecture de différents rapports qui émanent d'institutions internationales montre que cette question constitue l'une des figures du discours (OCDE, 2006; HCTE, 2007). Le rapport L'enseignement des sciences dans les établissements scolaires en Europe. Etat des lieux de la politique et de la recherche d'Eurydice publié en 2006 rappelle dans son préambule que «l'acquisition de compétences et de connaissances dans les matières scientifiques constitue un enjeu crucial pour l'Europe». En conséquence, de multiples voix, issues de la sphère politique comme du monde des scientifiques, se sont élevées pour souligner la nécessité d'améliorer l'enseignement des sciences à l'école. Ainsi l'enseignement des sciences fait-il lui-même l'objet de débats. Depuis les années 1990, des réformes ont été engagées dans un nombre important de pays. Notons que dans le cas qui nous intéresse, les réformes de l'enseignement des sciences ont eu pour toile de fond des situations très contrastées. Un exposé comparatif de l'évolution historique du système de chaque pays permettrait de se faire une représentation de la situation actuelle en inscrivant ces phénomènes dans une temporalité plus large. L'espace de cet article étant limité, nous nous risquerons donc à brosser à grands traits quelques éléments qui nous semblent distinctifs.

\section{Les récentes évolutions}

Comment caractériser les évolutions qui sont apparues? En République tchèque comme en Slovénie, la décennie des années 1990 a été une période de changements politiques et économiques majeurs et c'est dans ce contexte que l'évolution s'est produite. En 1991, un nouveau programme est publié en République tchèque. Il reprend les contenus et les méthodes qui étaient en vigueur dans les années précédentes ( $70 \%$ des thèmes du programme sont obligatoires et les $30 \%$ restant sont au libre choix des écoles). Il s'en démarque cependant, car il a été purgé de l'idéologie. En 2001 paraît un nouveau programme, le White Paper, qui va modifier de façon importante les bases de l'enseignement en République tchèque. En effet, il est pensé comme un documentcadre à partir duquel les écoles peuvent créer leur propre programme d'enseignement. Les réformes conduites en Slovénie l'ont été dans un contexte comparable. Rappelons que l'État slovène est reconnu depuis 1992 et qu'il s'agit donc d'un jeune État. L'indépendance a eu pour conséquence l'abandon du système socialiste et la mise en place d'un nouveau système économique. Dans ce contexte, le rôle des individus a été accentué et le cadre réglementaire des programmes a évolué : il ne s'agit plus de mémoriser de façon encyclopédique des faits, mais de construire des connaissances et des compétences. De nouveaux domaines comme les technologies de l'information sont apparus, le poids des évaluations internationales a été renforcé. Dès le début des années 1990, nos collègues slovènes ont tissé des liens avec d'autres partenaires 
d'Europe sur la question de l'enseignement des sciences. Dans ce cadre, ils ont organisé des séminaires de formation basés sur une approche constructiviste pour environ quatre cents enseignants du primaire. Ces enseignants ont à leur tour formé d'autres collègues grâce à un système en cascade. Les bons résultats obtenus par la Slovénie aux évaluations PISA et TIMSS (2007) montrent que le niveau des élèves s'est amélioré. Notons que dans ce pays, chaque année, les enfants bénéficient pendant au moins une semaine complète de «classes vertes» dans des structures adéquates, où ils peuvent mettre en ouvre des études de terrain, réaliser des travaux scientifiques et interdisciplinaires. Comme en Slovénie, les attendus des programmes irlandais $\left(\mathrm{NCCA}^{6}\right)$ sont basés sur des fondements socioconstructivistes. Dans ce pays, le milieu économique pèse pour que l'ensemble de la population dispose des savoirs scientifiques de base. Mais en Irlande, le poids des examens, à la fin du secondaire, semble trop grand. En théorie, ils ont été créés pour évaluer l'enseignement et permettre l'entrée dans l'enseignement supérieur. En réalité, ce sont eux qui pilotent l'enseignement secondaire, renversant ainsi les liens de causalité et illustrant l'expression "the tail that wags the dog» (la queue qui remue le chien).

D’une façon générale, on peut dire que les attentes des cadres réglementaires ont changé. La nécessité de proposer aux élèves des activités qui favorisent le développement des compétences est partout soulignée. Ces compétences sont pensées en calque de celles utilisées par les scientifiques dans leur travail de recherche. En outre, les enjeux culturels et citoyens de l'enseignement des sciences sont soulignés dans tous les programmes en vigueur. Suivant les pays, les descriptions des curricula peuvent varier mais il existe cependant des points de convergence forts. Ainsi, la majorité des programmes de l'enseignement secondaire inférieur font-ils référence à des activités qui exigent un «ensemble de connaissances et de savoir-faire complexes ainsi qu'une certaine autonomie des élèves ». On rencontre également cet élément, bien que dans une moindre mesure, dans les programmes de l'enseignement primaire (Eurydice, 2006). Sur ce dernier point, la situation en France semble moins clivée qu'ailleurs et l'on peut penser que les expériences La main à la pâte conduites depuis une dizaine d'années sont l'un des facteurs explicatifs de cette situation.

\section{Quelle formation des enseignants?}

Les rapports analysés pointent bien le rôle de la formation des enseignants, "principale interface entre les théories et la pratique de l'enseignement» (Eurydice 2006). Bien que les situations soient variables d'un pays à l'autre de l'Europe, il semble que la formation des enseignants du secondaire soit d'abord pensée sur un registre disciplinaire, ce qui «soulève des questions sur la façon dont les futurs enseignants sont armés pour mettre en place des approches inno-

6. NCCA : National Council for Curriculum and Assessment. Voir : http://www.ncca.ie/ [Consulté le 9 juin 2009]. 
vantes» (Eurydice, 2006). En outre, la mise en place en classe de situations pédagogiques ouvertes crée chez beaucoup d'enseignants un sentiment d'insécurité. Dès lors, il nous semble que la remarque du rapport Eurydice faite dans le cadre de la situation initiale des enseignants mérite d'être posée dans le cadre de la formation continue. Les enseignants plus expérimentés sont-ils armés pour mettre en place les situations ouvertes attendues par l'institution? Il nous a semblé que nous ne pouvions répondre par l'affirmative à cette dernière question.

Le but général du projet est de bâtir des modules de formation qui permettent aux enseignants de sciences de mettre en place dans leur classe des démarches qui «accompagnent la construction du savoir de l'élève». Ces modules ont été élaborés par les équipes de recherche des différents pays et ils ont été ensuite discutés et évalués de façon croisée.

\section{L'ÉLABORATION DE MODULES DE FORMATION EUROPÉENS}

\section{Modules identiques ou différenciés?}

Le groupe s'est trouvé confronté au dilemme suivant : allait-on produire des modules strictement identiques et les faire vivre sur le terrain dans les cinq pays partenaires? Ou allait-on jouer sur la diversité des approches et bâtir des modules différenciés pour chaque pays?

Choisir la première option offrait l'avantage de disposer d'un objet commun qui permettait une approche comparative. Cette option a été envisagée. Cependant, le simple fait de se trouver devant l'obligation de disposer d'un consensus a montré très vite les limites de cette approche. Comme nous l'avons vu, les situations dans les cinq pays engagés dans la recherche sont sensiblement différentes. L'analyse des organigrammes des différents systèmes éducatifs a montré des écarts, notamment pour ce qui est de l'enseignement secondaire. Le collège unique, spécificité française, les découpages disciplinaires différents et les paliers d'orientation nous ont permis de conclure que, si nous poursuivions le travail à la recherche d'un dénominateur commun, celui-ci risquait d'être très étroit. En effet, il existe dans certains pays un système de "cours accéléré» ou de «scolarité prolongée » pour les plus performants. En outre, une telle mise en œuvre offrait l'inconvénient de lisser les situations disponibles et de diminuer les écarts. Maintenir ce premier choix nous aurait ainsi privés d'une partie de la richesse de ce qui fait la diversité des pratiques éducatives en Europe. De plus se serait alors posé le problème de la diffusion et de la pertinence de nos modules. Nous avons donc choisi de travailler autrement. Les modules seraient bâtis en fonction des réalités locales pour répondre au mieux aux attentes des différents contextes, mais en prenant 
appui sur un cahier des charges commun. Ces modules seraient ensuite conduits et évalués (une évaluation interne, faite par l'équipe qui a élaboré le module et une évaluation croisée faite avec les autres partenaires). Actuellement, nous travaillons à pointer ce qui nous semble transférable d'un système à l'autre.

\section{Le fil conducteur commun retenu et ses appuis théoriques}

Comme il a été dit plus haut, nous avons choisi de nous inscrire dans un cahier des charges commun pour bâtir nos modules. La définition de notre cahier des charges provient du cadre théorique dans lequel nous nous inscrivons, qui nous conduit à appuyer les formations que nous souhaitons mettre en œuvre sur un modèle socioconstructiviste.

Dans cette perspective, faire un diagnostic des représentations et des savoirs antérieurs de l'élève, faire naître un conflit cognitif et/ou émotionnel et le soutenir, permettre que les élèves construisent une compréhension des phénomènes qu'ils abordent, aider l'élève à réfléchir à ses actions et encourager le transfert nous semblent être les compétences indispensables que les enseignants doivent posséder et qui doivent être développées grâce à nos modules.

Pour développer de telles attitudes, plutôt que d'élaborer des modules fondés sur un discours normatif et des méthodes à appliquer, nous avons choisi de faire vivre aux enseignants des situations plus ouvertes, de leur offrir un contexte d'apprentissage riche en interactions. Nous disposons actuellement de travaux qui montrent l'impact très limité des formations qui s'appuient sur une transmission d'informations (Wideen, Mayer-Smith et Moon, 1998). Par ailleurs, il semble établi que les apprentissages faits dans des contextes de formations qui s'appuient sur une faible participation des enseignants n'ont que peu de chance d'être transférés dans le quotidien de leurs classes (Fullan, 1993). Ainsi, l'un de nos objectifs est d'améliorer les capacités d'analyse réflexive des enseignants engagés dans ce processus.

\section{Actions conduites}

Dans trois des cinq pays (République tchèque, Slovénie et Irlande), les modules ont été conduits en choisissant des axes thématiques et sont indépendants les uns des autres. L'équipe française a centré son travail sur la mise en œuvre d'une démarche d'investigation. Les quatre modules ont été pensés comme appartenant à un tout et seul le premier module a été bâti à partir d'une entrée thématique. L'équipe de Chypre était dans une position intermédiaire et a travaillé des objets conceptuels ou des moments de la démarche.

Les équipes qui ont travaillé de façon thématique n'ont pas procédé de la même façon. Suivant les pays et les habitudes dominantes, il est plus ou moins facile d'obtenir une participation active des enseignants. Ainsi, nos 
collègues irlandais ont eu de réelles difficultés à obtenir une participation tangible des enseignants du secondaire. Certaines équipes se sont appuyées sur des matériaux existants, d'autres ont bâti entièrement des modules originaux.

Ne pouvant entrer dans une longue description des actions conduites, nous allons donc exposer brièvement l'état du travail.

L'équipe irlandaise a conduit quatre modules. Ont été explorés deux thèmes de physique ( forces et friction », "chute des corps dans l'air») et deux thèmes qui appartiennent aux sciences du vivant («comparaison d'écosystèmes", "évolution d'une population»). En République tchèque les thèmes explorés ont été «les mélanges», "la nutrition», «l'eau dans notre environnement» et les «cinq sens».

L'équipe de Chypre a travaillé trois thèmes; le quatrième est en cours. Ont été travaillés la notion d'hypothèse et le développement d'une argumentation scientifique à propos d'une expérience de combustion, la notion d'ombre et de lumière et la thématique «flotte ou coule».

L'équipe slovène a construit et conduit quatre modules. Les thématiques abordées ont été centrées sur des thèmes appartenant aux sciences du vivant. "Les plantes sont des êtres vivants» et "comment une plante se nourritelle?» ont constitué les thèmes des deux premiers modules. À la demande des enseignants, une monographie a été publiée pour diffuser plus largement ce travail. Les deux autres modules ont abordé la notion de circulation sanguine et de respiration. Un travail très fructueux a été proposé en incitant les enseignants à utiliser l'analogie comme moyen pédagogique avec leurs élèves.

En France, nous avons cherché à placer les enseignants dans un processus qui génère une dynamique durable et le projet a été pensé dans un cadre systémique. Quatre modules de formation de deux ou trois jours ont été répartis sur deux ans. Leur ensemble intègre des boucles de rétroaction qui se modifient en fonction de l'analyse des résultats obtenus. Par ailleurs, les enseignants ont accepté de concevoir et de mettre en ouvre des protocoles et des activités pour leurs classes, d'en rendre compte et d'en faire la relecture pour en tirer des logiques d'action. Dans ces modules des professeurs des écoles, des professeurs de physique chimie et des professeurs de sciences de la vie et de la terre en poste en collège ont travaillé ensemble. Nous avons choisi de constituer une population hétérogène qui comprend des professeurs confirmés et des professeurs débutants. Si d'autres pays ont choisi des entrées centrées sur des concepts ou des thèmes, nos choix se sont tournés vers la mise en œuvre de la démarche d'investigation et nous avons cherché à améliorer l'expertise et les pratiques des enseignants dans certaine certaines phases délicates de la démarche (conduite de débat en classe, la structuration du savoir) et à faire évoluer les représentations des enseignants à propos de la science. Lors du premier module, des thèmes («la respiration» et «ombre et lumière ») avaient 
été imposés. Nous avons par la suite laissé le choix des thèmes aux enseignants car cela permettait des essais en classe plus pertinents.

\section{Quelques pistes ouvertes par ce projet}

L'évaluation comparative de nos modules a ouvert des pistes. Ainsi, nous avons pu constater certaines réticences d'enseignants face à ce genre de démarches. Elles ont été plus particulièrement marquées dans certains pays pour les professeurs du secondaire (Irlande et République tchèque), alors que dans les mêmes pays, les enseignants du primaire n'ont pas manifesté de telles attitudes. Le fait que les programmes du secondaire soient souvent pensés dans une perspective encyclopédique et qu'il existe à ce niveau des évaluations très normatives n'est sans doute pas étranger à ce résultat.

À partir des travaux conduits par nos collègues slovènes, nous avons compris la nécessité de faire réfléchir les professeurs sur les critères qu'ils privilégiaient en classe et sur la nécessité de leur faire appréhender la différence entre une "observation» et une "prévision». L'expérience de nos collègues slovènes nous a aussi permis d'affiner notre réflexion. Dans ce pays, la pression conjuguée de la hiérarchie scolaire et des textes réglementaires a engendré ce que nos collègues ont décrit comme une "activitymania» (un certain activisme). Les enseignants mettent en ouvre dans leurs classes des expériences, des projets, des recherches, mais sans être toujours capables de définir clairement les objectifs qu'ils poursuivent ou d'établir des liens pertinents entre le faire et le prescrit. Cette dérive a aussi été constatée en France.

À partir des travaux conduits par nos collègues de Chypre, qui ont travaillé dans une perspective complètement constructiviste, nous avons perçu la nécessité de faire travailler les enseignants sur deux registres. D'une part, il semble indispensable de développer chez les enseignants des compétences en termes de conduite de classe, pour qu'ils laissent leurs élèves formuler leur propre questionnement. Mais d'autre part, il semble indispensable de leur faire percevoir qu'il n'est peut-être pas indispensable de tout déconstruire et qu'initier chez l'élève une démarche autonome ne signifie pas faire table rase de tout ce qui existe.

En France, dans la très grande majorité des cas, les enseignants ont conduit leurs séances et ont apporté des matériaux très riches. Les enseignants ont croisé leurs expériences et ont formulé une liste de points qui leur semblent particulièrement sensibles. Deux phases sont notamment délicates : celle qui consiste à permettre aux élèves de "s'approprier le problème» est difficile à conduire; de même, celle de la validation ou de la structuration du savoir nouveau en s'appuyant sur les expressions des élèves se révèle parfois ardue. Confrontés à des mises en situation issues de problématiques concrètes, les enseignants privilégient souvent un seul aspect du problème scientifique qui 
rend compte de cette réalité. Or une exploration plus systématique des possibles fait apparaître la complexité du réel sous-jacent; complexité que perçoivent souvent bien les élèves et qui se traduit dans leur questionnement, mais qui est tout aussi souvent peu entendue par les enseignants.

Ce point est un point de convergence. En particulier, nous avons pu constater les limites d'un système explicatif trop sommaire.

Pour exercer son métier, l'enseignant doit mobiliser une grande autonomie de pensée. La formation doit lui fournir un contexte et des moyens qui favorisent l'avènement de cette difficile conquête. C'est ce que nous avons cherché à favoriser dans la mise en œuvre de nos modules. Nos choix théoriques nous ont placés devant la nécessité de faire vivre de façon effective des situations aux enseignants, puis d'en faire avec eux des relectures. Leur vécu devient un élément déclenchant qui favorise ultérieurement la mise en ouvre de telles démarches dans le quotidien de leurs classes. Notre projet vise à engager les enseignants dans un processus qui devrait leur permettre de se placer dans une perspective de formation permanente. Dans quelle mesure cet objectif a-t-il été atteint? L'analyse fine des résultats, en cours, devrait permettre de le dire.

\section{RÉFÉRENCES}

RIC Report (2003) : National Exams Centre 2002/2003, Ljubljana : RIC.

CONVERT B. (2006) : Les impasses de la démocratisation scolaire. Sur une prétendue crise des vocations scientifiques. Paris : Editions Raisons d'agir.

EURYDICE (2006) : L'enseignement des sciences dans les établissements scolaires en Europe. État des lieux de la politique et de la recherche. Ce rapport est consultable sur le site : http://www.eurydice.org/.

ULE M. (2005) : CENYR. Voir : http://logic.itsc.cuhk.edu.hk/ b114299/reviews/ cenyr.pdf/.

PALEČKOVÁ J. (2007) : Hlavní zjištění výzkumu PISA 2006 - Poradí si žáci s přirodnimi vědami?, Ústav pro informace ve vzdělávání, Praha.

KANTORKOVÁ-LUKÁŠOVÁ H. (2004) : Příprava učitelů pro primární vzdělávání v ČR a budoucí plánování scénář̉ v Evropě. Pedagogická fakulta, Ostravské Univerzity, Ostrava.

BOILEVIN J.-M. \& RAVANIS K. (2007) : «L'éducation scientifique et technologique à l'école obligatoire face à la désaffection : recherches en didactique, dispositifs et références». Skholê, hors-série 1, 5-11. Consultable sur : http://recherche.aixmrs.iufm.fr/index.php ?som $=1 \& q u o i=$ sk\&lequel $=10 /$.

High Level Group on Science Education, ROCARD M. (Chair), CSERMELY P., JORDE D., LENZEN D., WALBERG-HENRIKSSON H., HEMMO V. (2007) : Science education now. A renewed pedagogy for the future of Europe. http://ec.europa. eu/research/rtdinfo/index_en.html. 
WIDEEN M., MAYER-SMITH J. et MOON B. (1998) : "A critical analysis of the research of learning to teach : Making the case for an ecological perspective on inquiry", Review of Educational Research, n², pp. 130-178.

FULLAN Michael, Change forces : probing the depths of educational reform. Philadelphia, Farmer Press, 1993.

NCCA, National Council for Curriculum and Assessment. Voir : http://www.ncca.ie [consulté le 9 juin 2009]. 